\title{
THE INFLUENCE OF VISUAL INFORMATION ON HABITUATION OF THE ELECTRODERMAL AND THE VISUAL ORIENTING REACTION
}

\author{
MARINUS N. VERBATEN, JACOB C. WOESTENBURG and WIM SJOUW \\ University of Utrecht, Department of Psychophysiology, University of Utrecht, Utrecht, \\ Holland
}

Accepted for publication 5 February 1979

In this study the influence of the information value of visual stimuli on habituation of the visual orienting reaction (VOR) and the skin conductance reaction (SCR) was investigated. 28 subjects received two blocks of 14 trials. Half the subjects received the higher information condition first and then the lower information, the other half received the conditions in the reversed order. Subjects fixated the stimuli with the higher information value longer than the stimuli with the lower information value during the 14 trials and habituated slower. This effect of information was absent in the second block and VOR habituation was also faster in this block. There was no difference in SCR amplitudes nor in SCR habituation scores between the two information conditions, neither in block 1 nor in block 2 . The results of this study are discussed in relation with a two-stage model of the OR.

\section{Introduction}

According to Sokolov (1963), stimuli with a high information value should induce larger initial orienting reactions (OR's) and should be more resistant to habituation than stimuli with low information value. Lovibond (1969) defined information in terms of stimulus uncertainty and found significant effects of information on habituation rate of the skin conductance reaction (SCR). However, Or and Stern (1969) found no evidence of the influence of information on SCR habituation using a similar procedure. O'Gorman (1971) presented series of letters with different information values (1,2 and 3 bits) to his subjects and found significant effects on the SCR habituation rate. In a later study (O'Gorman, 1973), however, it was pointed out that a SCR is not necessarily an index of the recognition of a stimulus or recognition of a change of stimulation, because some subjects although able to report a stimulus change accurately, show no SCR. Spinks and Siddle (1976), defining stimulus information in terms of stimulus complexity (Attneave, 1954), found that stimuli with higher information value induced slower SCR habituation, but did not induce larger initial SCR's. The question, therefore, whether or not information value of the stimulus influences the OR and habituation of the OR has not been settled. In this study we investigated the effect of stimulus information 
not only on the SCR component of the OR, but also on the visual orienting reaction (VOR), a behavioral component of the OR, which consists of the turning of the eyes towards the source of stimulation. It is generally assumed (Kahneman, 1973, p. 56) that eye-movements towards the source of stimulation are beneficial for the processing of information. Mackworth and Bruner (1970) showed that those parts of the visual field that contained more information also attracted more fixations. Leeuwenberg (1967) showed that stimuli containing more information induced longer spontaneous fixation times, using a new definition of information in terms of stimulus complexity. In several experiments habituation of the VOR was investigated. Mackworth and Otto (1970) showed a decrease of fixation during habituation and Furst (1971). showed an increasing stereotype in the behavior of eye movements during habituation, which manifested itself in a more global and less accurate inspection of the stimulus. The behavior of eye movements towards a stimulus source can thus be used as an index of the OR.

Some preliminary remarks on our approach to the VOR in this study are necessary. We measured the eye movements in reaction to stimuli which were presented in the periphery of the visual field, viz. at a $28^{\circ}$ angle from the centre. Sanders (1963, p. 48) showed that under these conditions subjects have to make a saccadic eye movement for accurate perception of the stimulus. We expected that these saccadic eye movements would show habituation after,enough information about the stimulus has been processed and thus lead to a decrease of total fixation time of the stimulus. We also expected that stimuli with a higher information value would induce longer initial fixation times.

As all the subjects in this experiment received the two information conditions, half the subjects in one order and the other half in the reversed order, we could investigate whether or not habituation during the first condition affects habituation in the second condition and whether or not there are differences between the two $O R$ indices in this regard, provided any of the OR indices are in fact influenced by the information value of the stimulus.

\section{Method}

\subsection{Subjects}

Subjects were 28 undergraduate students of psychology at the University of Utrecht, 10 males and 18 females. Mean age 20.7, s.d. 5.18 Subject wearing glasses kept them on during the experiment.

\subsection{Apparatus}

\subsubsection{EOG}

Standard $\mathrm{Ag}-\mathrm{AgCl}$ EEG electrodes in plastic cups were used for the electro- 
oculogram (EOG). The electrodes were placed at the outer canthus of each eye for the horizontal EOG. Infra-orbital and supra-orbital electrodes were placed in line with the pupil of the left eye for the vertical EOG. Standard EEG paste was used. Resistance of the electrodes was never higher than $2 \mathrm{k} \Omega$. The signals from the electrodes were amplified and filtered ( $\mathrm{RC}$ time was $36 \mathrm{~s}$ ) by an Elema universal filter. The long RC time was chosen to cut off slow changes caused by polarization effects of the electrode--electrolyte combination.

Amplifier output was first sent through a $50 \mathrm{~Hz}$ notch filter and then sent to analogue inputs of the PDP 11/40 computer for on line analogue-digital conversion and scoring. Sample rates were $40 \mathrm{~Hz}$. Sampling during calibration started $240 \mathrm{~ms}$ after stimulus onset and lasted $200 \mathrm{~ms}$. Sampling during habituation trials and during the control procedure started at stimulus onset and lasted $2 \mathrm{~s}$.

\subsubsection{SCR}

The SCR was measured by a constant voltage system $(0.5 \mathrm{~V})$. Two $\mathrm{Ag}-\mathrm{AgCl}$ pellet electrodes (diameter $0.5 \mathrm{~cm}$ ) were attached to the palm of the left hand. The electrode paste contained a 0.05 molar concentration of $\mathrm{NaCl}$ in a agar-agar base. Conductance values were transformed into digital values and then sent to the digital input of the PDP $11 / 40$.

Sample frequency of these digital values was $5 \mathrm{~Hz}$. Sampling lasted from $0.5 \mathrm{~s}$ to $6 \mathrm{~s}$ after stimulus onset. Scoring was done by the PDP $11 / 40$.

\subsection{Stimulus conditions}

During the experiment subjects lay on a examination table in an acoustically and electrically shielded room. The upper portion of the examination table was adjustable, so that the subject's head could be adjusted to a position parallel to a television monitor (black-white, 26 inch screen), which was positioned above and in front of the subject, at a distance of $60 \mathrm{~cm}$ from the subject's eyes. Stimuli and instructions were presented to the subjects on this TV monitor. Clamps were used to position subject's head so that the centre of the TV screen was in the centre of the visual field. Eye movements from the centre of the TV screen to any of the four corners of the screen were 28 degrees of arc. Stimuli presented during the calibration period were the letters $X$ in the four corners of the screen and the letter $O$ in the centre of the screen. During calibration the duration of these stimuli was $1.5 \mathrm{~s}$. Intervals between presentations were $3 \mathrm{~s}$.

During habituation trials the stimuli containing the two information values were presented in the upper right corner (see fig. 1) for a period of $2.0 \mathrm{~s}$. The length of intervals between stimulus presentations during habituation trials were randomly chosen and varied between $15 \mathrm{~s}$ and $25 \mathrm{~s}$.

Eye-movements and SCR's as well as marks of stimulus onset and offset were also displayed as ink-records on a Mingograph 80 polygraph. Horizontal and vertical EOG's were also displayed on a $\mathrm{X}-\mathrm{Y}$ oscilloscope for purposes of checking. 
Presentation of stimuli and of instructions and sampling procedures were controlled by the PDP $11 / 40$.

\subsection{Procedure}

On arrival the subjects were familiarized with the procedure and put at ease regarding the methods employed and their part in the experiment. After the electrodes were attached in the instrument room, which adjoined the experimental room, the subject lay down on the examination table and the instruments were connected. The head was fixed in the desired position and subject was informed that instructions would follow on the TV screen. The experiment started with a 5 -min adaptation period, after which the instructions were presented. These included a request to move as little as possible and not to fall asleep. Eye movement calibration then commenced.

\subsubsection{Calibration}

First the letter $O$ was presented in the centre of the screen. Then at regular intervals the letter $O$ was followed by the letter $X$ in each of the four corners of the screen. The subject was asked to fixate the letters as accurately as possible. The first trial was used to familiarize the subject with the calibration procedure. The second time EOG's were sampled and the computer was programmed to construct a two-dimensional representation of the four different positions of the letters $\mathrm{X}$ as perceived by the subject.

In order to measure the accuracy of the eye movements towards the stimuli during the habituation trials, the computer was programmed to construct two circles of decreasing accuracy around the stimulus that was to be presented in the upper right corner during habituation trials. The inner circle (field I) scored maximal accuracy of the eye movements towards these stimuli and the outer circle (field II) scored the least accurate eye movements (see fig. 1).

This calibration procedure was repeated before the second block of 14 trials.

\subsubsection{Habituation}

After the calibration procedure the subjects were instructed that a number of figures would be presented on the TV screen. They were told that they did not have to react in any special way to these stimuli. Thereafter each subject received one block of 14 trials in which figures with 12 bits of information were presented and one block of 14 trials in which the figures with 60 bits information were presented. Half the subjects received first the block with 12 bits and then the block with 60 bits. The other half received the reversed order. All figures were presented in the upper right corner of the TV screen. 


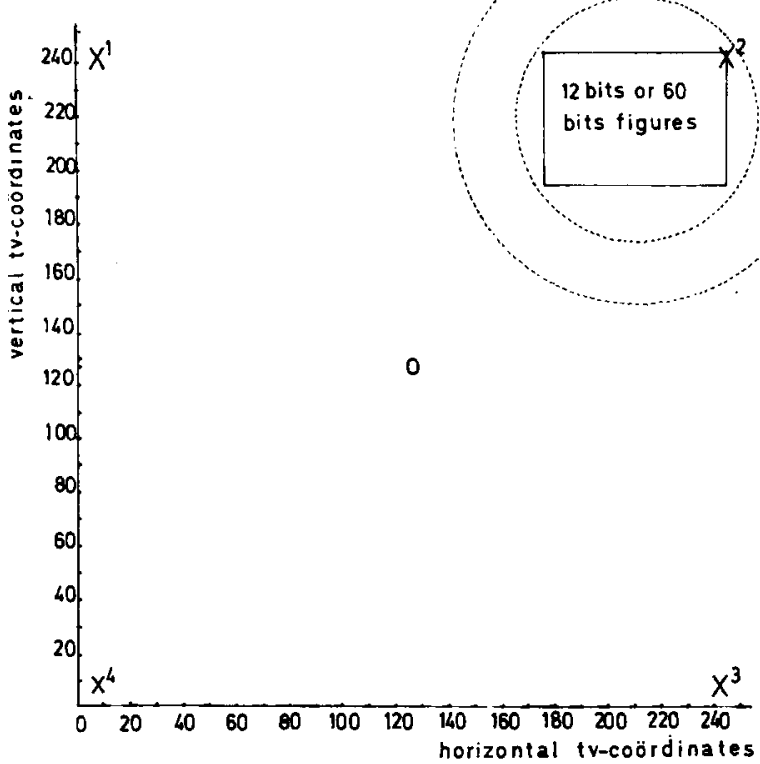

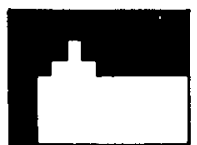

12 bits

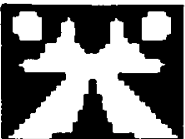

60 bits

Fig. 1. Distances of the four calibration stimuli $\left(\mathrm{X}^{1}, \mathrm{X}^{2}, \mathrm{X}^{3}, \mathrm{X}^{4}\right)$ and the information containing stimulus (inf) in TV coordinates $(256 \times 256)$. The discontinuous lines indicate the two windows field $I$ and field II around the information containing stimulus, which is presented during the habituation trials. Horizontal distance is $\mathbf{4 5}$ degrees of arc, vertical distance is 36 degrees of arc.

\subsubsection{Check}

After each block of 14 trials there was a checking procedure. The subjects were told that a different figure (on precisely the same TV coordinates as the habituation stimuli) would be presented. They were asked to fixate this new figure accurately. These fixations were scored with the same program as was used for scoring the VOR's. In this way it could be determined whether changes in eye movement behavior during the habituation trials were real or determined by artefacts like head movement or drift.

At the end of the experiment the subjects were given two simple recognition tests, one for the 12 bits figure and one for the 60 bits figure. Subjects had to indicate the correct figure among three slightly different ones.

\subsection{Scoring}

\subsubsection{SCR}

Each change in conductance larger than $0.01 \mu \mathrm{mho}$, occurring within $0.5-6 \mathrm{~s}$ after stimulus onset was scored as an OR and transformed into a delta conductance 
square root score. Delta conductance is the difference between the peak of amplitude of the skin conductance reaction and the pre-reaction conductance level. Scoring was done on line with a computer program. Reactions lasting shorter than 600 $\mathrm{ms}$, from beginning to peak, were not scored as an OR, SCR habituation scores were the number of SCR's occurring till the habituation criterion of three consecutive responses of less than $0.01 \mu$ mho was reached.

\subsubsection{VOR}

Fixation criterion was $100 \mathrm{~ms}$ of either a fixation inside field I or field II. The VOR was the sum of the fixations inside these fields in $\mathrm{ms}$.

The VOR habituation score was the number of VOR's till the habituation criterion of three consecutive zero responses was reached.

\section{Results}

The repeated measurements are treated in the analysis as multiple intercorrelated responses of the same subjects. This means that the assumptions about the nature of the repeated measurements data are the usual ones in multivariate models. A multivariate analysis of variance was performed (Finn, 1976) with order of presentation of conditions as a between-subjects factor and trials and information as within-subjects factors. For the effects of trials and information a 5\% one-tailed level of significance was set because it was expected that the OR indices would decrease over trials and that the stimulus with the higher information value would lead to larger initial reactions and slower habituation than the figure with the lower information value. As there were no specific expectations in regard to the factor of order and the interaction effects, a 5\% two-tailed level of significance was set in those cases.

The results of the analysis are summarized in table 1 .

Table 1

Summary of the multivariate analysis; univariate $F$ 's and df's for the three dependant variables field I, field II and SCR

\begin{tabular}{llclc}
\hline Source & df & Field I & Field II & SCR \\
\hline Order $(O)$ & 1.26 & 1.47 & 0.40 & 0.05 \\
Information $(I)$ & 1.26 & $\left.5.47^{\mathrm{a}}\right)$ & 0.10 & 0.62 \\
Trials $(T)$ & 1.26 & $13.67^{\mathrm{c}}$ & 0.23 & $\left.11.96^{\mathrm{c}}\right)$ \\
$O \times T$ & 1.26 & 0.10 & 0.01 & 0.44 \\
$O \times I$ & 1.26 & $12.53^{\mathrm{c}}$ & 0.10 & $\left.5.65^{\mathrm{a}}\right)$ \\
$I \times T$ & 1.26 & 0.01 & 0.01 & 0.33 \\
$O \times I \times T$ & 1.26 & 0.92 & 0.02 & 0.41 \\
\hline
\end{tabular}
a) $p<5 \%$ one-tailed
b) $p<5 \%$ two-tailed
c) $p<1 \%$ two-tailed 
The main effect of trials was significant for field I and for the SCR. Field II did not show a significant effect of trials which means that it did not show habituation over trials, one of the most fundamental characteristics of the OR.

The main effect of Information was significant for field I of the VOR but not for the SCR and field II. As far as field II is concerned this lead to the conclusion that field II could not be considered to be part of a visual OR as it did not show
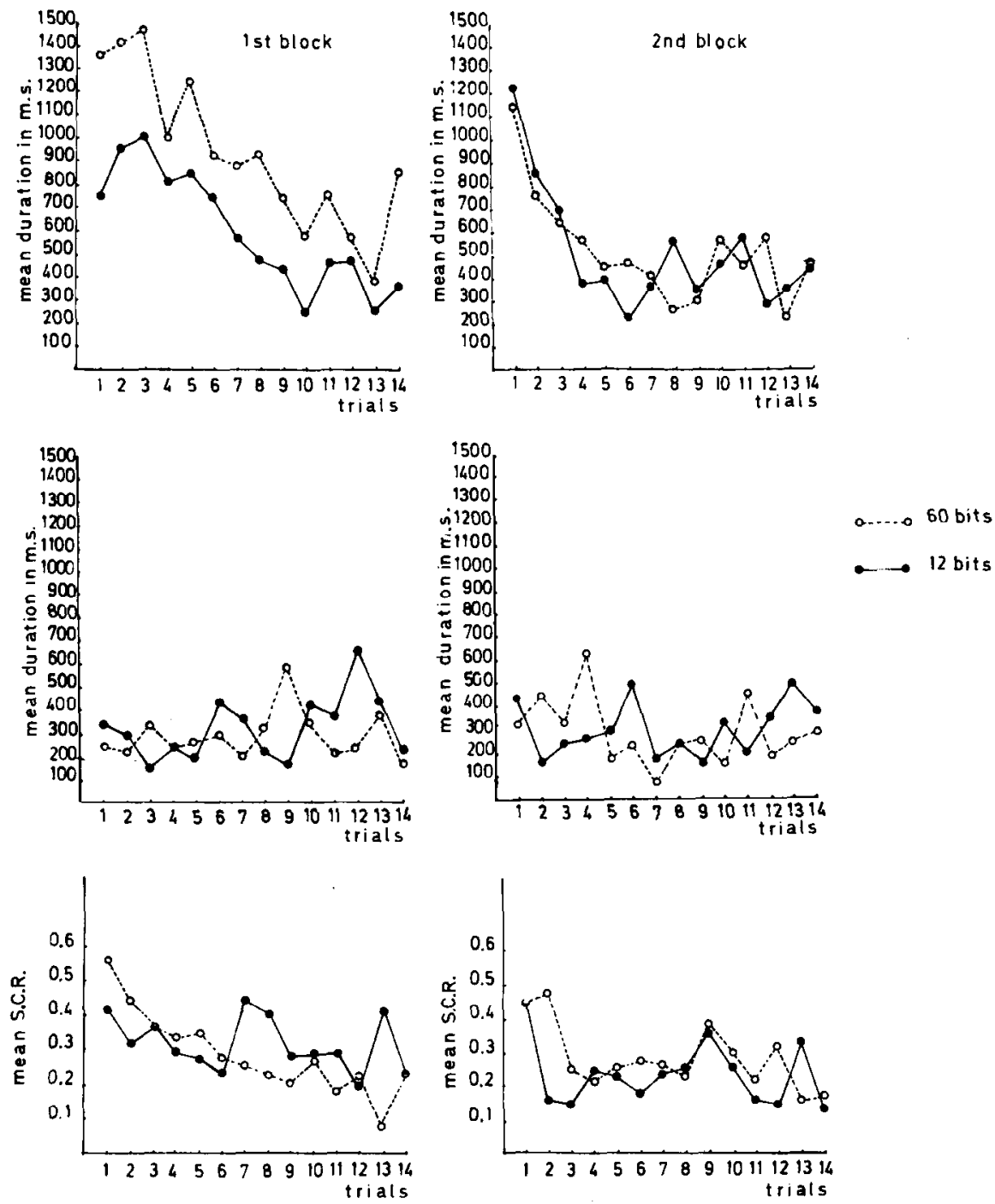

Fig. 2. Mean fixation times scored in field I, field II and SCR during block 1 and block 2. Solid line: 12 bits condition, discontinuous line: 60 bits condition. 


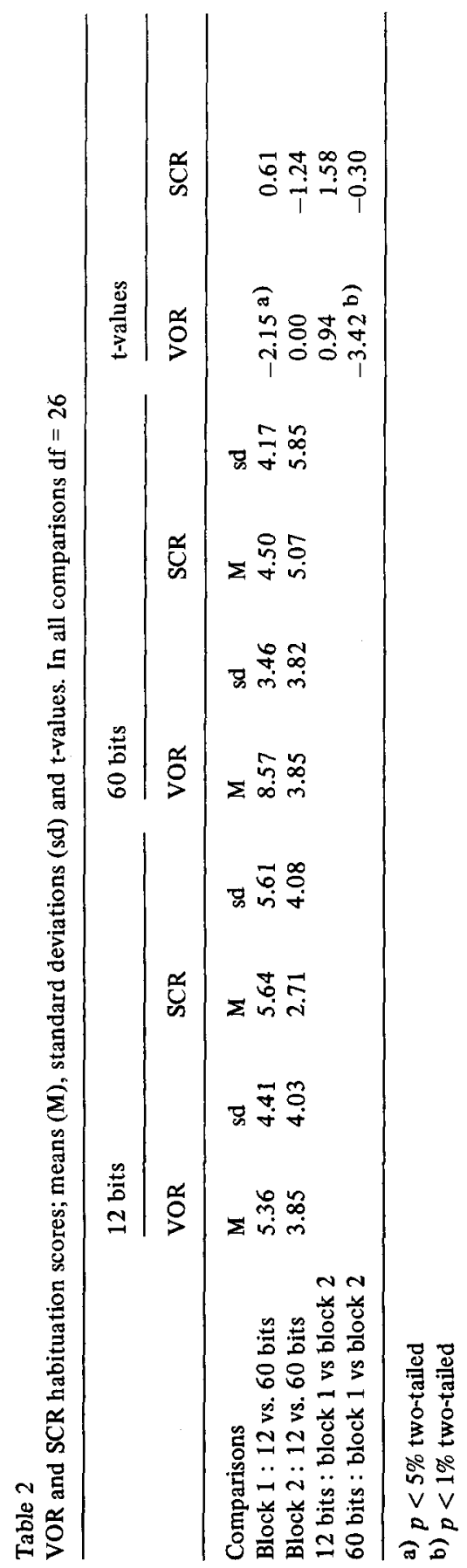


habituation and was not sensitive for differences in information value of the stimuli in this experiment. On the other hand this indicated that field I was a reasonably good approach to the visual $O R$ as this field showed both habituation and reflected the differences in information value. There were no main effects of the factor Order, but field I of the VOR showed a significant order $\mathrm{X}$ information interaction which is illustrated in fig. 2. All the other interactions were not significant. The significant effect of information was only seen during the first block and the information effect disappeared during the second block.

We also tested whether the conditions of this experiment had effects on the SCR and VOR habituation scores. In order to do so, we first had to redefine the VOR as the total duration of fixations of minimally $100 \mathrm{~ms}$, falling in field $\mathrm{I}$, because fixations in field II did not qualify as an OR. We determined with the $t$-test for independent samples (Ferguson, 1959) whether there were differences between the two information conditions during block 1 and block 2 . The results are summarized in table 2 .

During block 1 VOR habituation scores were significantly higher during the 60 bits information condition than during the 12 bits information condition. No significant effects were found for the VOR habituation scores during block 2, nor for the SCR habituation scores during block 1 and block 2 .

We also tested with the $t$-test for independent samples whether the habituation scores under the two information conditions were lower during the second block. The only significant difference was in regard to the 60 bits condition; the VOR habituation score during the second block was significantly lower than during the first block.

In order to show that changes in fixation time over trials (habituation) were not caused by artefacts, trials 1,14 , and the check trial were tested against each other with the Wilcoxon matched pairs rank test (Siegel, 1956, pp. 42-75).

A score of 2 was given when the subject fixated in field I, score 1 to fixations in field II and a score of 0 was given for fixations outside this fields.

The results are summarized in table 3 .

The results in table 3 indicate that there were no significant differences between the check trial and trial 1, while these trials differed significantly from trial 14 .

Table 3

Results of the Wilcoxon matched pairs rank test; $z$ values and two-tailed $p$ values

\begin{tabular}{lllll}
\hline & \multicolumn{2}{l}{ Conditions } & \\
\cline { 2 - 4 } & 12 bits & & 60 bits \\
\hline Comparisons & $z$ & $p$ & $z$ & $p$ \\
Trial 1-14 & -3.07 & $1 \%$ & -2.55 & $5 \%$ \\
Trial 14-check & -2.46 & $5 \%$ & -2.63 & $1 \%$ \\
Trial 1-check & -0.42 & ns & -0.84 & ns \\
\hline
\end{tabular}



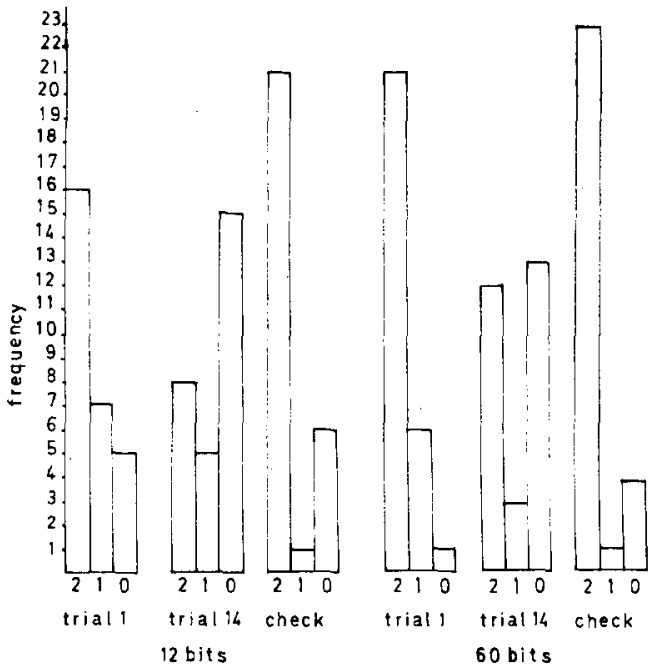

Fig. 3. Number of subjects (frequency) which fixated during trial 1 , trial 14 and the check trial within field I, field II or outside these fixation fields: (a) indicates the results for the 12-bits condition; (b) indicates the results for the 60-bits condition.

Subjects recognized at the end of the experiment significantly better the 12 bits figure than the 60 bits figure (resp. 24 and 12 correct recognitions) as indicated by the Chi-square test for dependent samples (Siegel, 1956, pp. 42-75) $\left(\mathrm{X}^{2}=9.60\right.$, $\mathrm{df}=1, p<1 \%$ two-tailed).

\section{Discussion}

Saccadic eye movements towards a visual stimulus in the periphery of the subjects' visual field showed habituation when that stimulus was presented repeatedly. The VORs during the 14 trials lasted significantly longer when a figure with 60 bits information was presented, than when a 12-bits figure was presented, which is in accordance with results of Leeuwenberg (1967) and Mackworth et al. (1970) who also found that fixation time and eye movements were influenced by the complexity of visual stimuli. That the 60-bits figure actually contained more information was also illustrated by the fact that recognition scores at the end of the experiment for the 60-bits figure were significantly lower than for the 12-bits figure; it was a more difficult figure to remember.

Restating the VOR-findings from this experiment in Sokolov's (1963) terms, we can conclude that stimuli with a higher value of information induced larger OR's. But this conclusion can only be drawn for the VOR and not for the SCR, as we found no significant difference in SCR-amplitudes between the two information- 
conditions. In this respect there was a difference between the two OR-indices. It appeared that there was a asymmetrical effect (Poulton and Freeman, 1966) in the balanced design used in this study. The difference between the two information conditions was only present in the first of the two blocks. This was also the case with the VOR-habituation scores. During the first block the VOR habituation scores were significantly higher in the 60-bits condition. In the second block there was no difference between the two information conditions. As with the SCR amplitudes there was no difference between the SCR habituation scores of the two information conditions, neither during block 1 , nor during block 2 . Not only was the information-effect absent in block 2, VOR-habituation took also place faster; VORhabituation scores during the 12 bits and 60 bits conditions were lower during block 2 (in the 60-bits condition statistically significant). A possible explanation for this effect might be that the subjects were less uncertain about the situation during the second block; during the second block the position of the stimulus, stimulus duration and ISI's were the same as during the first block. This suggests that stimuli containing more visual information were only fixated longer when the subjects were uncertain about the experimental situation; when this uncertainty was reduced, complexity had a less strong effect on the VOR. This suggestion is at variance with Velden (1978) who concluded 'the strength of the OR was found to be a function of the information given by the stimulus (stepwise completion of a sentence) independent of the residual entropy'. It is clear that this two different suggestions are based upon different experimental designs and different psychophysiological parameters, so no straightforward comparison can be made. Research is in progress to investigate the relationship between uncertainty and complexity during habituation.

Spinks and Siddle (1976) found that SCR-habituation during the 60-bits condition was significantly slower than during the 12-bits condition. The difference between these results and the results in our study might be explained by the fact that they instructed their subjects to pay attention to the stimuli while we did not.

According to Kahneman (1969) electrodermal activity is higher when the subjects show more mental effort. It might be that the task-relevance condition (Spinks and Siddle, 1976) induced more mental effort during the more difficult condition (60 bits), leading to more SCR activity.

Bagshaw, Kimble and Pribam (1965) suggested that the SCR is not involved in the production of orienting activity (= attention) directly but in its registration. As far as registration can be interpreted as 'further processing' after an initial phase of the OR, both Bagshaw et al.'s (1965) and Kahneman's (1967) hypotheses concerning electrodermal activity lead to the suggestion that electrodermal activity is associated with a secondary stage in the OR process. The VOR might be associated with a more primary phase of the OR, during which information about the situation is collected. Information collected during this initial phase does not necessarily have to be processed in the secondary stage. This is suggested by the fact that Spinks and Siddle (1976) not only found slower SCR habituation during the 60-bits condition, 
indicating higher electrodermal activity, but also reported that their recognition task was successfully completed by all subjects. In our experiment we found neither. It might be that during this secondary stage further processing of the stimuli might lead to more stable memory traces and that the SCR activity is associated with that process. The suggestion is offered with the necessary precaution because the task in the experiment of Spinks and Siddle (1976) was almost certainly easier; their subjects received only one information condition, while our subjects received two information conditions, probably causing interferention to some extent. Research is in progress to investigate the relation between stimulus information and task relevance during habituation.

\section{References}

Attneave, F. (1954). Some informational aspects of visual perception. Psychological Review, 61, 183-193.

Bagshaw, M.H., Kimble, D.P. and Pribram, K. (1965). The GSR of monkeys during orienting and habituation and after ablation the amygdala hippocampus and inferotemporal cortex. Neuropsychologia, 3, 111-119.

Ferguson, G.A. (1959). Statistical Analysis in Psychology and Education. McGraw-Hill: New York.

Finn, J.D. (1976). Multivariance, Version V, Release 3: A Fortran IV Program. National Educational Resources: Chicago.

Furst, C.J. (1971). Automatizing of visual attention. Perception and Psychophysics, 10, 65-69.

Kahneman, D. (1969). Pupillary, heart rate and skin resistance changes during a mental task. Journal of Experimental Psy chology, 79, 164-167.

Kahneman, D. (1973). Attention and Effort. Prentice Hall: New-Jersey.

Leeuwenberg, E.L. (1967). Structural Information of Visual Patterns. Mouton: Paris and The Hague.

Lovibond, S.H. (1969). Habituation of the orienting response to multiple stimulus sequences. Psychophysiology, 5, 435-439.

Mackworth, N.H. and Bruner, J.S. (1970). How adults and children search and recognize pictures. Human Development, 13, 149-177.

Mackworth, N.H. and Otto, D.A. (1970). Habituation of the visual orienting response in young children. Perception and Psychophysics 7, 173-180.

O'Gorman, J.G. (1971). Habituation of the orienting reaction as a function of stimulus information. Psychonomic Science, 22, 331-332.

O'Gorman, J.G. (1973). Change in stimulus conditions and the orienting response. Psychophysiology, 10, 465-470.

Or, W.C. and Stern, J.A. (1969). The effect of stimulus information on habituation rate. Paper presented at the Annual Meeting of the Society for Psychophysiological Research, Monterey, California.

Poulton, E.C. and Freeman, P.R. (1966). Unwanted asymmetrical transfer effects with balanced experimental designs, Psychological Bulletin, 66, 1-8.

Sanders, A.F. (1963). The Selective Process in the Functional Visual Field. Van Gorcum: Assen.

Siegel, S. (1956). Non-parametric Statistics for the Behavioral Sciences. McGraw-Hill: New York. 
Sokolov, E.N. (1963). Perception and the Conditioned Reflex. Pergamon Press: Oxford.

Spinks, J.A. and Siddle, D.A.T. (1976). Effects of stimulus information and stimulus duration on amplitude and habituation of the electrodermal orienting response. Biological Psychology , 4, 29-39.

Velden, M. (1978). Some necessary revisions of the neuronal model concepts of the orienting response. Psychophysiology, 15, 181-185. 